\title{
Quantum Causality Threshold and Paradoxes
}

\author{
Florentin Smarandache, Ph D \\ Chair of Math \& Sciences Department \\ University of New Mexico \\ 200 College Road, Gallup, NM 87301, USA
}

\begin{abstract}
:
In this paper we consider two entangled particles and study all the possibilities: when both are immobile, or one of them is immobile, or both are moving in different directions, or one of them is moving in a different direction. Then we study the causality between them and the paradoxes, which are generated. We define the Causality Threshold of a particle A with respect to another particle B.
\end{abstract}

Keywords: entangled particles, causality, causality threshold, quantum paradoxes

\section{Perfect simultaneousness.}

Let's consider two entangled particles A and B. \{Schrödinger introduced the notion "entangled" in order to describe the non-separable states [Belavkin (2002)]\}.

At the beginning, both are immobile, in the same space $\mathrm{S}(\mathrm{A}, \mathrm{B})$ and time $\mathrm{t}$ (simultaneously), and none of them is in the causality cone of the other.

According to Einstein's Theory of Relativity, when a particle is moving with respect to the other, its time and space axes appear inclined from the perspective of the other particle, modifying what for this other particle is "before" or "after", but their causality cones remain the same. And, if both particles are moving with respect to each other, the appearance of the inclined time and space axes is reciprocal from the perspective of each other. 
Let's define the Quantum Causality Threshold of the particle A with respect to the particle $\mathrm{B}$, noted by $\tau_{\mathrm{A}, \mathrm{B}}$, to be the space-time when neither $\mathrm{A}$ nor $\mathrm{B}$ is a cause for the other on the B space-time axis (i.e. when the position-time vector vertex $\left.t_{\mathrm{A}} \equiv \mathrm{B}\right)$.

To change the causality of a particle A with respect to another particle B one has to pass through non-causality, i.e. one has to pass through their threshold.

Generally, $\tau_{A, B} \neq \tau_{B, A}$, because one can have $t_{A} \equiv B$ but $t_{B} \neq A$, or reciprocally [see, for example, Figure 1.1.1].

a) When $\tau_{\mathrm{A}, \mathrm{B}}=\tau_{\mathrm{B}, \mathrm{A}}$ there is no causality between $\mathrm{A}$ and $\mathrm{B}$ (and therefore there is no quantum causality paradox).

b) If one particle attains its threshold with respect to the other, and the other one does not, then there is a causality and a non-causality simultaneously (and thus a quantum causality paradox) [see Figures $1.1 .1,1.1 .2,1.2 .1]$.

c) If no particle attains its threshold with respect to the other, one has two sub-cases: either opposite causalities (and thus, again, a quantum causality paradox) [see Figures 1.1.3, 1.1.4], or compatible causalities (and, consequently, there is no quantum causality paradox) [see Figures 1.2.2 (for $t$ together with $t$ time axes), Figure 1.2.3 (for $t$ together with t'" time axes)].

\subsection{Moving particle(s) keeping the same direction.}




\subsubsection{Particle B is moving away from particle A}

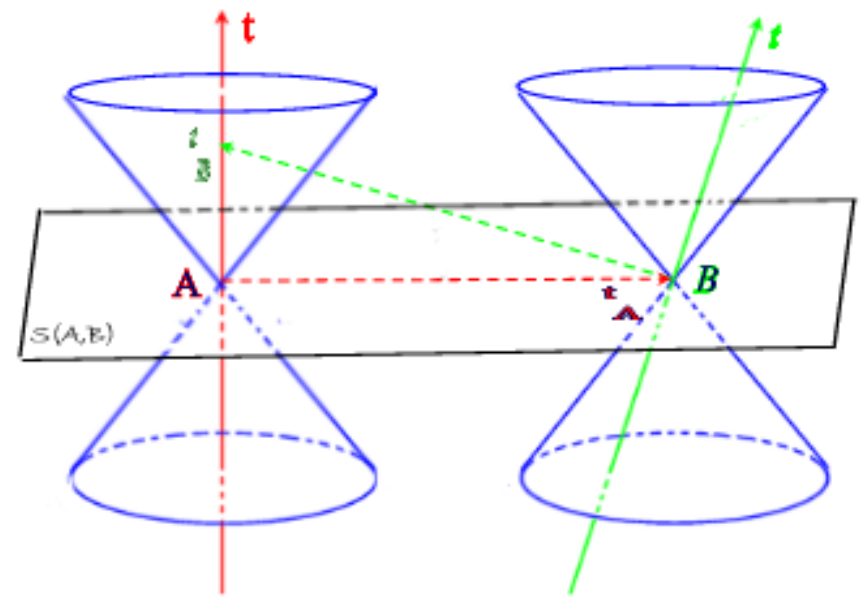

Figure 1.1.1-

- $\mathrm{S}(\mathrm{A}, \mathrm{B})$ is the space (represented here by a plane) of both entangled particles A and B .

- The left red vertical ( $t$ ) continuous line represents the time axis of the particle A.

- Similarly, the green ( $t$ ) continuous line represents the time axis of the particle B.

- On the left side one has the double cone of causality of the particle A: the cone beneath $\mathrm{S}(\mathrm{A}, \mathrm{B})$ contains the events that are the cause for $\mathrm{A}$ (i.e. events that influenced $A$ ), and the cone above $S(A, B)$ contains the events that $\mathrm{A}$ is a cause for (i.e. events influenced by A).

- Similarly, the right double cone represents the cone of causality of the particle B.

- Beneath $\mathrm{S}(\mathrm{A}, \mathrm{B})$ it is the past time ("before $\mathrm{A}$ "), lying on the $\mathrm{S}(\mathrm{A}, \mathrm{B})$ is the present time ("simultaneously with $A$ "), and above $\mathrm{S}(\mathrm{A}, \mathrm{B})$ it is the future time ("after A").

- Similarly, because the particles A and B are in the same space, $S(A, B)$ separates the past, present, and future times for the particle B. 
Relative to the same referential system, the particle A remains immobile, while the particle B starts moving in the opposite direction relative to A. [Figure 1.1.1]

Therefore, from the perspective of $\mathrm{B}$, the entangled particles $\mathrm{A}$ and $\mathrm{B}$ are simultaneous, and none of them is the cause of the other $\left(t_{\mathrm{A}} \equiv \mathrm{B}\right.$ on $\mathrm{B}$ 's time axis); while from the perspective of $\mathrm{A}$, the particle $\mathrm{A}$ is a cause for the particle $B$ (i.e. $A<t_{B}$ on $A$ 's time axis).

Hence, it appears this quantum causality paradox: non-causality or causality simultaneously?

\subsubsection{Particle B is moving closer to particle A}

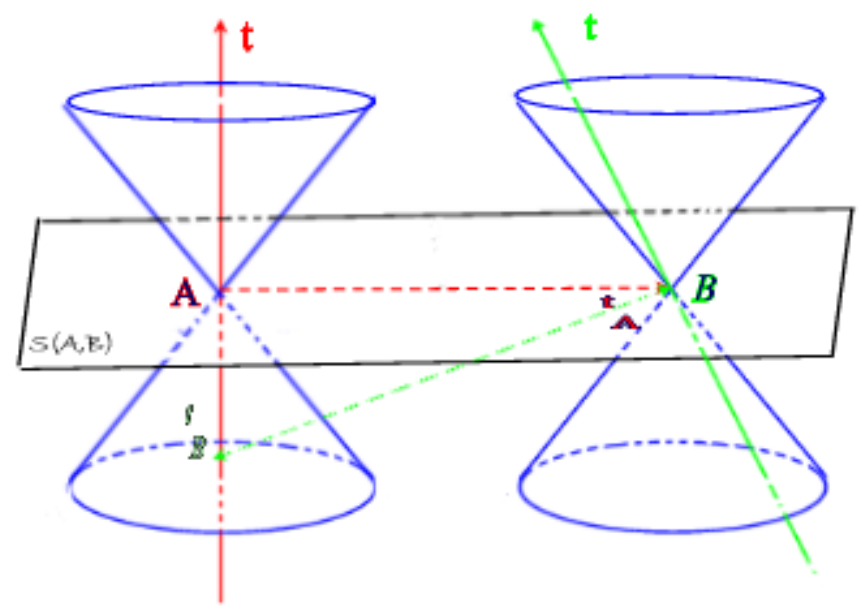

Figure 1.1.2

Relative to the same referential system, the particle A remains immobile, while the particle B starts moving in a direction towards A. [Figure 1.1.2]

Therefore, from the perspective of the particle $\mathrm{B}$, the entangled particles $\mathrm{A}$ and $\mathrm{B}$ are simultaneous, and none of them is the cause of the other $\left(t_{A} \equiv B\right.$ on B's time axis); while from the perspective of the particle $\mathrm{A}$, the particle $\mathrm{B}$ is a cause for the particle A (i.e. $\mathrm{tB}<\mathrm{A}$ on A's time axis).

Hence, again, it appears a similar quantum causality paradox: non-causality or causality simultaneously? 


\subsubsection{Both entangled particles are moving closer to each other}

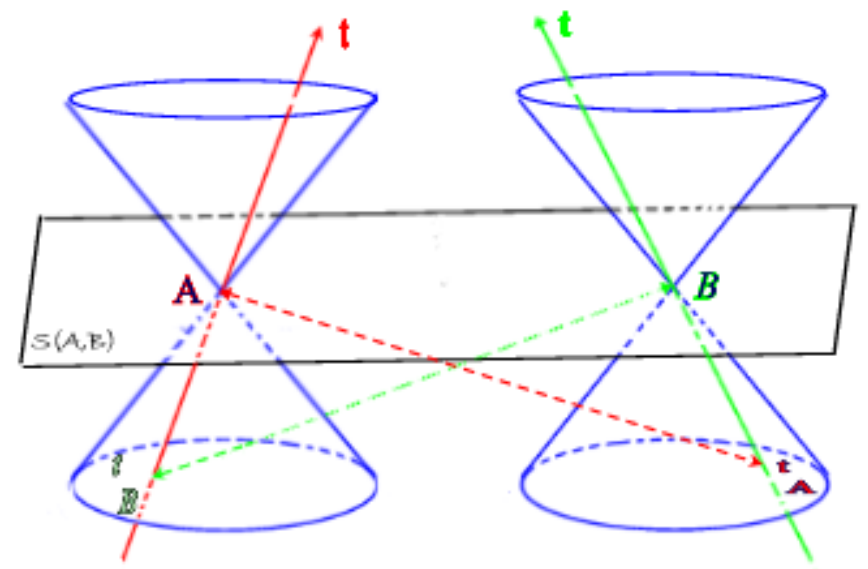

Figure 1.1.3

With respect to the same referential system, both particles A and B start moving towards each other. [Figure 1.1.3]

Therefore, from the perspective of the particle A, the particle B is a cause of the particle $A$ (i.e. $t_{B}<A$ on A's time axis), and reciprocally: from the perspective of the particle $B$, the particle $A$ is a cause of the particle $B$ (i.e. $t_{A}<$ $\mathrm{B}$ on B's time axis). Thus one obtains the following:

Quantum Causality Paradox: How is it possible that simultaneously A is a cause of $\mathrm{B}$, and $\mathrm{B}$ is a cause of $\mathrm{A}$ ? 


\subsubsection{Both entangled particles are moving away from each other}

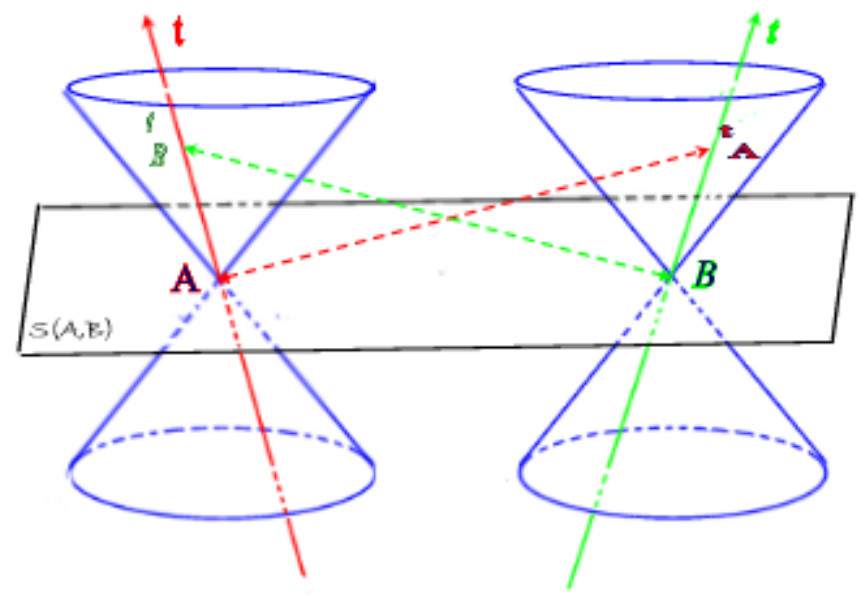

Figure 1.1.4

With respect to the same referential system, both particles A and B start moving in opposite directions from each other. [Figure 1.1.4]

Therefore, from the perspective of $\mathrm{A}$, the particle $\mathrm{A}$ is a cause of the particle $\mathrm{B}$ (i.e. $A<t_{B}$ on $A$ 's time axis), and reciprocally: from the perspective of $B$, the particle $B$ is a cause of the particle A (i.e. $B<t_{A}$ on B's time axis). Thus, one obtains the following same statement:

Quantum Causality Paradox: How is it possible that simultaneously A is a cause of $\mathrm{B}$, and $\mathrm{B}$ is a cause of $\mathrm{A}$ ?

This theoretical case is similar to the 2002 Suarez Experiment [1], the only difference being that in Suarez's experiment there is not a perfect simultaneousness between the particles A and B.

\subsection{Moving particle(s) changing the direction.}

1.2.1. With respect to the same referential system, the particle A is immobile; while the particle $\mathrm{B}$ is moving at the beginning in a direction towards $\mathrm{A}$, and later B changes the direction moving away from $\mathrm{A}$. 
a) Then, from the perspective of $A$ : The particle $B$ is a cause for $A$ (i.e. $t_{B}<A$ on A's time axis). Then $B$ changes its movement in a direction away from $\mathrm{A}$, consequently $\mathrm{B}$ attains its quantum threshold $\tau_{\mathrm{B}, \mathrm{A}}$, i.e. $\mathrm{t}^{\prime}{ }_{\mathrm{B}}$ $\equiv \mathrm{A}$ on A's time axis (now there is no causality between A and B). B keeps moving further from $A$ and crosses its quantum threshold, then $A$ becomes a causality for B because $\mathrm{t}^{\prime}{ }_{\mathrm{B}}>\mathrm{A}$ on A's time axis.

b) While, from the perspective of $\mathrm{B}$, there is no causality between $\mathrm{A}$ and $B$, since $B \equiv t_{A}$ on all $B$ 's three time axes $t^{\prime}, t^{\prime \prime}, t^{\prime \prime}$. [Figure 1.2.1.]. Hence, this quantum causality paradox appears: simultaneously $\mathrm{B}$ is cause for $\mathrm{A}$, and non-causality, and $\mathrm{A}$ is cause for $\mathrm{B}$ ?

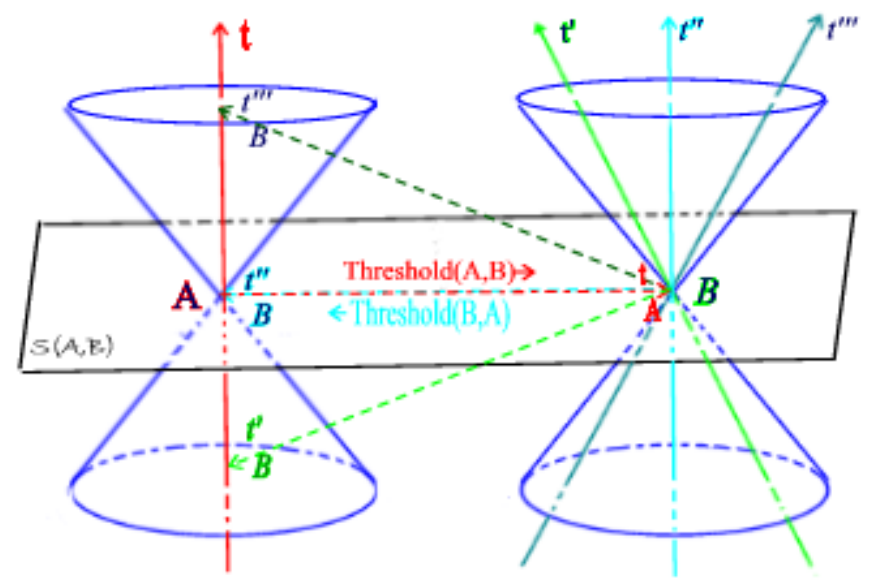

Figure 1.2.1

1.2.2. Relative to the same referential system, the particle A is moving away from $\mathrm{B}$; while the particle $\mathrm{B}$ is moving at the beginning in a direction towards $\mathrm{A}$, and later $\mathrm{B}$ changes the direction moving away from $\mathrm{A}$.

a) Then from the perspective of $\mathrm{A}$ : $\mathrm{B}$ is a cause for $\mathrm{A}$ (i.e. $\mathrm{t}_{\mathrm{B}}<\mathrm{A}$ on $\mathrm{A}$ 's time axis). Then $B$ changes its movement in a direction away from $A$, consequently $\mathrm{B}$ attains its quantum threshold $\tau_{\mathrm{B}, \mathrm{A}}$, i.e. $\mathrm{t}_{\mathrm{B}} \equiv \mathrm{A}$ on $\mathrm{A}$ 's 
time axis (now there is no causality among A and B). B keeps moving further from $\mathrm{A}$ and crosses its quantum threshold, then $\mathrm{A}$ becomes a causality for B because $t^{\prime \prime}>A$ on A's time axis.

b) While from the perspective of $\mathrm{B}$, the particle $\mathrm{B}$ is always a cause for A, since $\mathrm{B}<\mathrm{t}_{\mathrm{A}}$ on all $\mathrm{B}$ 's time axes $\mathrm{t}^{\prime}, \mathrm{t}^{\prime}$, and $\mathrm{t}^{\prime \prime}$. [Figure 1.2.2]. Hence, this quantum causality paradox appears: simultaneously $\mathrm{B}$ is cause for $\mathrm{A}$, and non-causality, and $\mathrm{A}$ is cause for $\mathrm{B}$ ?

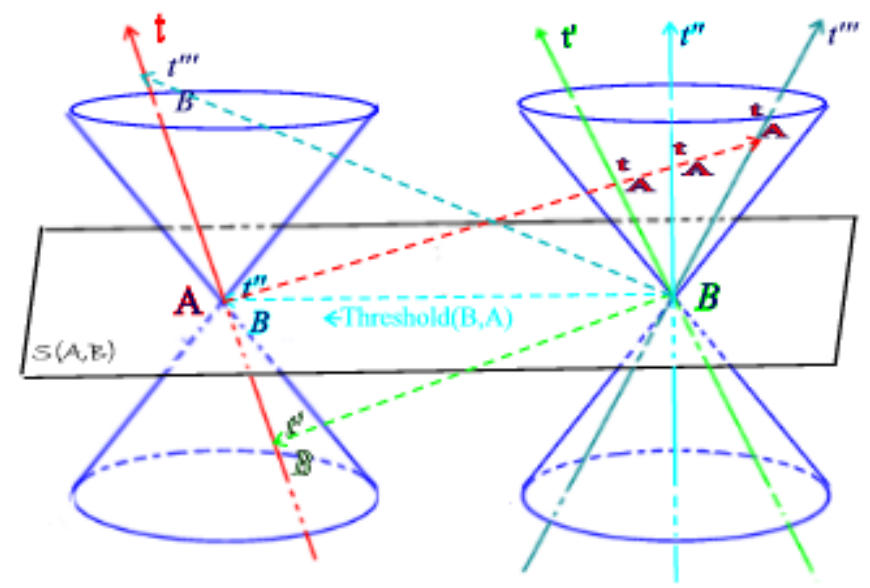

Figure 1.2.2

1.2.3. With respect to the same referential system, the particle A is moving closer to $\mathrm{B}$; while the particle $\mathrm{B}$ is moving at the beginning in a direction towards $\mathrm{A}$, and later $\mathrm{B}$ changes the direction moving away from $\mathrm{A}$.

a) Then from the perspective of A: B is a cause for A (i.e. $\mathrm{t}_{\mathrm{B}}<\mathrm{A}$ on $\mathrm{A}$ 's time axis). Then $B$ changes its movement in a direction away from $A$, consequently B attains its quantum threshold $\tau_{B, A}$, i.e. ${ }^{\prime \prime}{ }_{B} \equiv \mathrm{A}$ on A's time axis (now there is no causality among $\mathrm{A}$ and $\mathrm{B}$ ). $\mathrm{B}$ keeps moving further from $\mathrm{A}$ and crosses its quantum threshold, then $\mathrm{A}$ becomes a cause for $B$, because $t^{\prime}{ }_{B}>A$ on A's time axis. 
b) While from the perspective of $\mathrm{B}$, the particle $\mathrm{A}$ is always a cause for $\mathrm{B}$, since $t_{A}<B$ on all $B$ 's time axes $t$, $t^{\prime \prime}$, and $t^{\prime \prime}$. [Figure 1.2.2]. Hence, this quantum causality paradox appears: simultaneously $B$ is cause for $\mathrm{A}$, and non-causality, and $\mathrm{A}$ is cause for B?

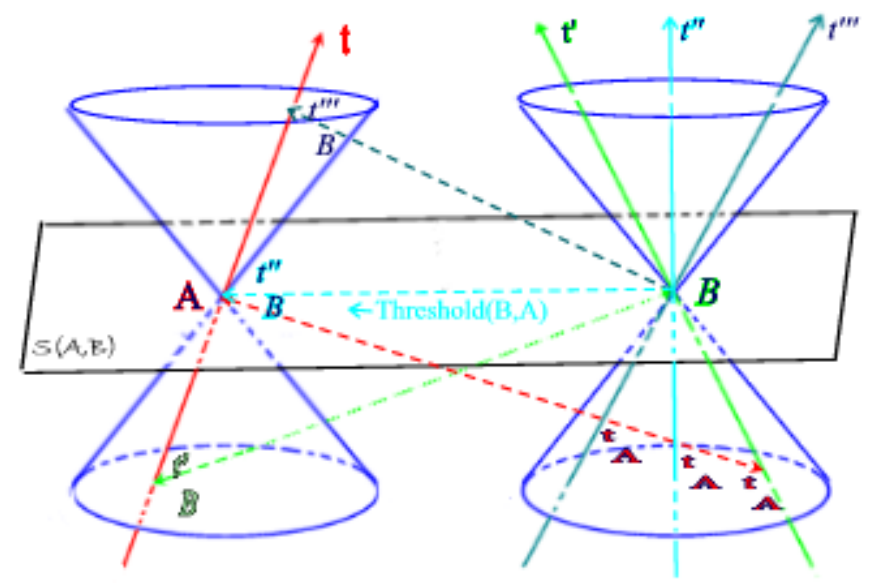

Figure 1.2.3

2. Let's consider the non-simultaneousness, when the particles $A$ and $B$ are in the separate spaces, $\mathrm{S}(\mathrm{A})$ and $\mathrm{S}(\mathrm{B})$ respectively, and different time axes, $t$ and $t$ 'respectively.

\subsection{Moving particle(s) keeping the same direction.}

2.1.1. With respect to the same referential system, both particles A and B are moving in the same direction but with different high speeds. [Figure 2.1.1] Therefore, from both perspectives, of $\mathrm{A}$ and of $\mathrm{B}$, the particle $\mathrm{B}$ is cause for $\mathrm{A}$. 


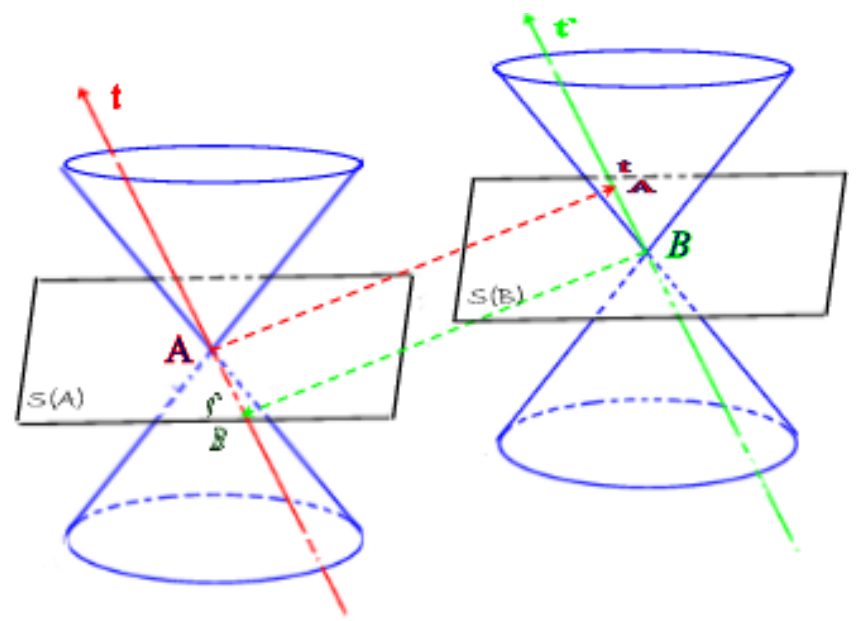

Figure 2.1.1

2.1.2. With respect to the same referential system, both particles $A$ and $B$ are moving in the same direction and with the same high speeds. [Figure 2.1.2] Therefore, from both perspectives, of A and of B, neither one is the causality of the other. 


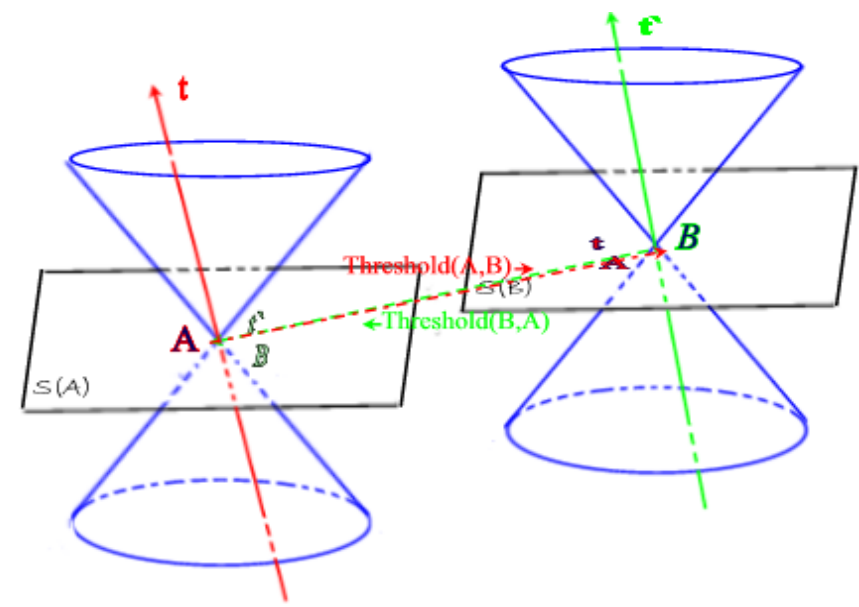

Figure 2.1.2

2.1.3. With respect to the same referential system, both particles A and B are moving closer to each other and with different high speeds [Figure 2.1.3]. Therefore, from the perspective of $\mathrm{A}$ the particle $\mathrm{B}$ is a cause of $\mathrm{A}$, and reciprocally, thus again one gets a quantum causality paradox.

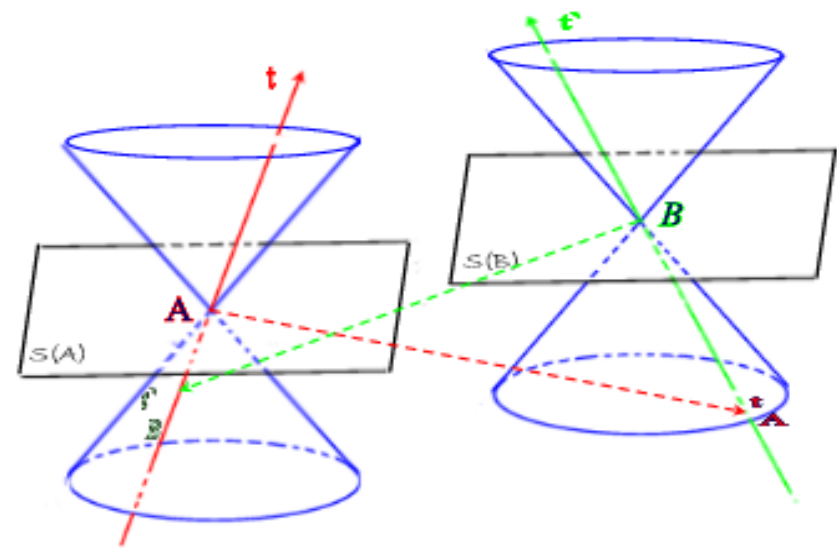

Figure 2.1.3 


\subsection{Moving particle(s) changing the direction.}

2.2.1. With respect to the same referential system, the particle $A$ is moving towards $\mathrm{B}$; while the particle $\mathrm{B}$ is moving at the beginning in a direction towards $\mathrm{A}$, and later $\mathrm{B}$ changes the direction moving away from $\mathrm{A}$.

a) Then from the perspective of A: B is a cause for A (i.e. $\mathrm{t}_{\mathrm{B}}<\mathrm{A}$ on $\mathrm{A}$ 's time axis). Then $B$ changes its movement in a direction away from $A$, consequently $\mathrm{B}$ attains its quantum threshold $\tau_{\mathrm{B}, \mathrm{A}}$, i.e. $\mathrm{t}_{\mathrm{B}} \equiv \mathrm{A}$ on $\mathrm{A}$ 's time axis (now there is no causality among $\mathrm{A}$ and $\mathrm{B}$ ). $\mathrm{B}$ keeps moving further from $\mathrm{A}$ and crosses its quantum threshold, then $\mathrm{A}$ becomes a cause for B because $\mathrm{t}^{\prime \prime}{ }_{\mathrm{B}}>\mathrm{A}$ on A's time axis.

b) While from the perspective of $\mathrm{B}$, the particle $\mathrm{A}$ is always a cause for $\mathrm{B}$, since $t_{A}<B$ on all B's time axes $t^{\prime}, t^{\prime \prime}$, and $t^{\prime \prime}$. [Figure 2.2.1.]. Hence, this quantum causality paradox appears: simultaneously B is cause for $\mathrm{A}$, and non-causality, and $\mathrm{A}$ is cause for $\mathrm{B}$ ?

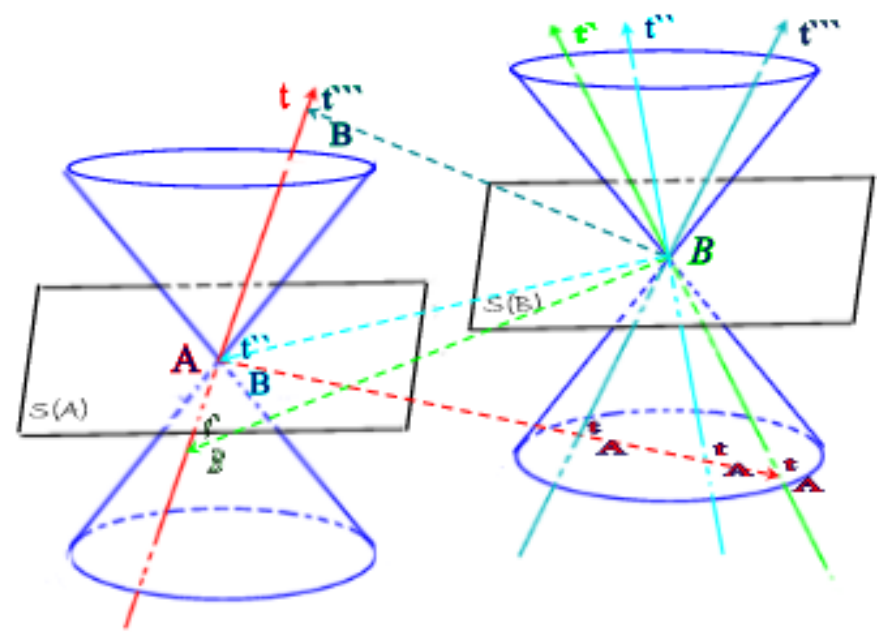

Figure 2.2.1 
2.2.2. Relative to the same referential system, both particles are moving towards each other, and then both change the movement in the opposite directions.

Similarly, from both perspectives, of A and of B, there are normal causalities (corresponding to $\mathrm{t} 1$ and $\mathrm{t}^{\prime}$ time axes), non-causalities (corresponding to $\mathrm{t} 2$ and $\mathrm{t}$ " time axes), and opposite causalities (corresponding to $\mathrm{t} 3$ and $\mathrm{t}$ " " time axes) [Figure 2.2.2].

Hence, one again, one arrives at quantum causality paradoxes.

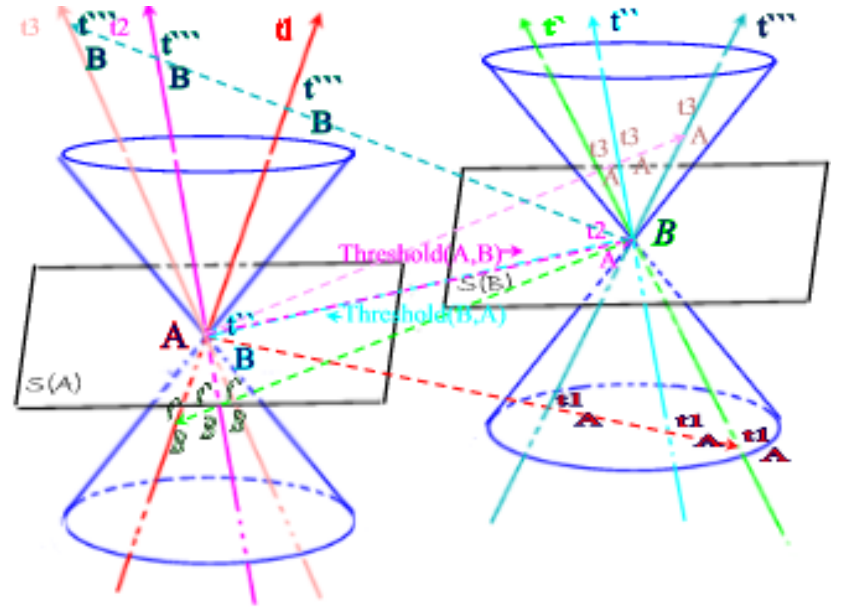

Figure 2.2.2 


\section{Acknowledgement:}

I would like to thank Dr. Jean Dezert from ONERA, France, who made me aware of this paradox of the time and provided me the Poirier's article.

\section{References:}

1. Belavkin, V. P., "Quantum Causality", Decoherence, Trajectories and Information, arXiv:quant-ph/0208087 v2, 2002.

2. Poirier, Hervé, “Le Temps N'Existe Pas!”, Science \& Vie, Paris, No. 1024, 36-50, 2003.

3. Smarandache, Florentin, "There is no speed barrier in the Universe", Bulletin of Pure and Applied Science, 17D (Physics), No. 1, p. 61, 1998, http://www.gallup.unm.edu/ smarandache/NoSpLim.htm.

4. Gilbert, John, "What is your opinion on Smarandache's hypothesis that there is no speed barrier in the universe?", Ask Experts (Physics): http://www.physlink.com/ae86.cfm.

5. Illingworth, Valerie, editor, "Dictionary of Physics", The Penguin, London, New York, Victoria, Toronto, 1990.

6. Le, Charles T. Le, " 'There Is No Speed Barrier in the Universe' ", book review, The Internet Pilot TO Physics, http://physicsweb.org/TIPTOP/FORUM/BOOKS.

7. Smarandache, Florentin, Collected Papers, Vol. III, Abaddaba Publ. Hse., Oradea, Romania, 158, 2000.

8. Smarandache, Florentin, Cultural Tour to Brazil on "Paradoxism in Literature and Science": "Is There a Speed Barrier?", Universidade de Blumenau, May 31 - June 20, 1993.

9. Smarandache, Florentin, "Definitions, Solved and Unsolved Problems, Conjectures, and Theorems in Number Theory and Geometry", edited by M. Perez, 86 p., Xiquan Publ. House, Phoenix, 73, 2000.

10. Smarandache, Florentin, "Life at Infinite Speed", Arizona State University, Hayden Library, Special Collections, Tempe, USA, 1972.

11. Smarandache, Florentin, "Neutrosophic Logic and Superluminal Speed even Infinite Speed", University of Kishinev, Scientific Conference, chaired by 
Professors Gheorghe Ciocan, Ion Goian, and Vasile Marin, University of Kishinev, December 1994.

12. Smarandache, Florentin, "There Is No Speed Barrier in the Universe", $<$ Bulletin of Pure and Applied Sciences $>$, Delhi, India, Vol. 17D (Physics), No. 1, p. 61, 1998.

13. Smarandache, Florentin, "There Is No Speed Barrier in the Universe", http://www.gallup.unm.edu/ smarandache/NoSpLim.htm, http://www.geocities.com/m 1 perez/SmarandacheHypothesis/Sm-Hyp.htm (in Quantum Physics online journal).

14. Suplee, Curt, " 'Beaming Up' No Longer Science Fiction", <Albuquerque Journal>, December 11, 1997.

15. Walorski, Paul (A. B. Physics), Answer to J. Gilbert, Ask Experts: http://www.physlink.com/ae86.cfm.

16. Weisstein, Eric, "Smarandache Hypothesis", Eric Weisstein's World of Physics, Wolfram Research, USA,

http://scienceworld.wolfram.com/physics/SmarandacheHypothesis.html. 